\title{
Closed-Form Solution to Disambiguate Defocus Blur in Single-Perspective Images
}

\author{
Majed El Helou Marjan Shahpaski Sabine Süsstrunk \\ School of Computer and Communication Sciences, EPFL, Switzerland
}

\begin{abstract}
Depth-from-defocus techniques suffer an ambiguity problem where depth planes on opposite sides of the focal plane have identical defocus. We solve the ambiguity by relying on the wavelength-dependent relationship between defocus and depth. We conduct a robustness analysis and validation on consumer lenses. (C) 2019 The Author(s)
\end{abstract}

OCIS codes: $080.1010,120.5710$.

\section{Mathematical Framework and Solution}

Our proposed solution is developed in a simple lens framework. Blur is characterized by the radius of a circle of confusion, representing the image of a point light source, and is wavelength dependent. For a wavelength channel $C$, the blur radius is $r_{C}(d)=L\left|1-x / f_{C}+x / d\right|$, where $L$ is the simple lens aperture radius, $x$ is the distance between the center of the lens and the sensor plane, $f_{C}$ is the lens' focal length for channel $C$ and $d$ is the depth of the source point relative to the lens. For a given channel $C$ and sensor position, the focal plane is at depth $d_{C}^{0}$, at which the blur radius is minimal: $r_{C}\left(d_{C}^{0}\right) \rightarrow 0$. This depth is given by $d_{C}^{0}=x\left(x / f_{C}-1\right)^{-1}$. Varying the depth $d$, under a realistic scenario where $x>f_{C}$, the blur radius is a convex function of $d$, with a minimum at the focal plane. This creates an ambiguity that was solved recently using coded-aperture lenses [1] or calibrated and memorized for modified lenses over shallow pre-defined depth ranges [2]. Here, we leverage the differential blur across spectral channels. We consider two channels $A$ and $B$ of different wavelength and set w.l.o.g. $f_{B}>f_{A}$. Noticing that the blur radius is wavelength-dependent, we study the measure $\Delta_{B, A}(d) \triangleq r_{B}(d)-r_{A}(d)$ given by

$$
\Delta_{B, A}(d)= \begin{cases}\alpha \triangleq L\left(x / f_{A}-x / f_{B}\right) & d \leq d_{A}^{0} \\ 2 L(1+x / d)-L\left(x / f_{A}+x / f_{B}\right) & d \in\left[d_{A}^{0}, d_{B}^{0}\right] \\ -\alpha=L\left(x / f_{B}-x / f_{A}\right) & d \geq d_{B}^{0} .\end{cases}
$$

For $d$ smaller than $d_{A}^{0}$ or larger than $d_{B}^{0}, \Delta_{B, A}(d)$ is constant and only depends on the camera parameters $(L$ and $x$ ) and the focal lengths of the two spectral channels $A$ and $B$. In practice, $f_{A}$ and $f_{B}$ are close between RGB channels, making the depths $d_{A}^{0}$ and $d_{B}^{0}$ close, which in turn decreases $\alpha$. Also, complex lenses are designed to correct color chromatic aberration and minimize the shift between color focal planes. Sec. 3 shows that the shift is nevertheless detectable even with complex lenses, and that with near-infrared (NIR) the corresponding $\alpha$ value becomes noticeably larger, allowing for a more robust solution (Fig. 1).

The $\Delta$ measure can be estimated directly from an input image. For a given image patch $I(d)$, captured at wavelength $\lambda_{C}$, we observe a blurred version $I_{b}\left(d, \lambda_{C}\right)=I(d) * P S F_{e q}\left(d, \lambda_{C}, u, v\right)=I(d) * H_{d e f}\left(d, \lambda_{C}, u, v\right) * H_{0}(u, v)$, where $d$ is the depth of the object in pixel coordinates $(u, v)$. $H_{d e f}$ represents defocus blur in channel $C$, and $H_{0}$ all wavelength-invariant blur (e.g., motion blur). The variance of $P S F_{e q}$ is $\sigma_{e q}^{2}\left(d, \lambda_{C}, u, v\right)=\sigma_{d e f}^{2}\left(d, \lambda_{C}, u, v\right)+$ $\sigma_{0}^{2}(u, v)$. When the blur radius $r_{C}(d)$ is estimated, $\sigma_{e q}^{2}\left(d, \lambda_{C}, u, v\right)$ is approximated instead of the desired $\sigma_{d e f}^{2}\left(d, \lambda_{C}, u, v\right)$, modifying the estimated value of $r_{C}(d)$ by an offset shift [3]. As $\sigma_{0}^{2}(u, v)$ is invariant with respect to wavelength, the measure $\Delta_{B, A}(d)$ defined as $\sigma_{d e f}^{2}\left(d, \lambda_{B}, u, v\right)-\sigma_{d e f}^{2}\left(d, \lambda_{A}, u, v\right)$, can be estimated by the pixelwise subtraction $\sigma_{e q}^{2}\left(d, \lambda_{B}, u, v\right)-\sigma_{e q}^{2}\left(d, \lambda_{A}, u, v\right)$ that cancels the undesirable offset. The sign of this estimated $\Delta_{B, A}(d)$ is sufficient to resolve the blur ambiguity, and obtain a bijective blur-depth mapping.

\section{Error Bounds Analysis for the Measure $\Delta$}

Channels capture radiation across a wavelength range determined by the sensor's color filter array. Looking at the intensity present in a channel $C$, the captured radiation may have any wavelength $\lambda_{C} \pm \delta_{C}$ where $\lambda_{C}$ is the central wavelength and $\delta_{C}$ is a wavelength shift bound to the filter limits. In the extreme case, all radiation captured in the channel $C$ has wavelength $\lambda_{C}+\delta_{C}^{\max }$ instead of $\lambda_{C}$, where $\delta_{C}^{\max }$ is the maximum deviation. $\delta_{C}^{\max }$ corresponds to a shift $\gamma_{C}^{\max }$ in focal length. We also denote by $e_{C}$ the algorithmic blur estimation error, and obtain the predicted blur 

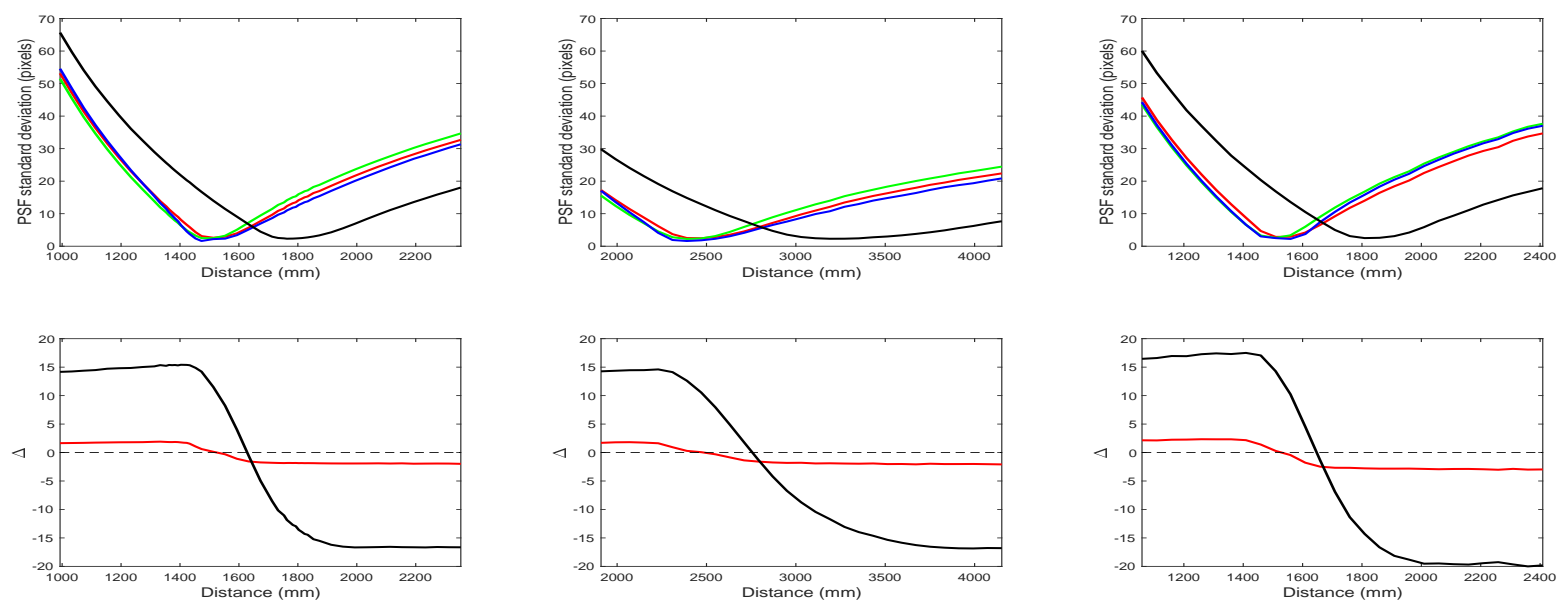

Fig. 1. Top: blur magnitude vs depth for the RGB (color) and NIR (black) channels, on 3 image sets. Blur is estimated as the standard deviation of a Gaussian fitted to the edge spread function, computed on a $5^{\circ}$ ISO 12233 edge. Bottom: corresponding $\Delta$ plots for $\Delta_{R, G}$ (red) and $\Delta_{N I R, G}$ (black).

radius $r_{C}^{\prime}(d)=L\left|1-\frac{x}{f_{C}+\gamma_{C}^{m a x}}+\frac{x}{d}\right|+e_{C}$. The resulting difference $\Delta_{B, A}^{\prime}(d) \triangleq r_{B}^{\prime}(d)-r_{A}^{\prime}(d)$ becomes

$$
\Delta_{B, A}^{\prime}(d)= \begin{cases}L\left(\frac{x}{f_{A}+\gamma_{A}^{\max }}-\frac{x}{f_{B}+\gamma_{B}^{\max }}\right)+E_{B, A} & d \leq d_{A}^{0^{\prime}} \\ 2 L\left(1+\frac{x}{d}\right)-L\left(\frac{x}{f_{A}+\gamma_{A}^{\max }}+\frac{x}{f_{B}+\gamma_{B}^{\max }}\right)+E_{B, A} & d \in\left[d_{A}^{0^{\prime}}, d_{B}^{0^{\prime}}\right] \\ L\left(\frac{x}{f_{B}+\gamma_{B}^{\max }}-\frac{x}{f_{A}+\gamma_{A}^{\max }}\right)+E_{B, A} & d \geq d_{B}^{0^{\prime}},\end{cases}
$$

where $E_{B, A} \triangleq e r_{B}-e r_{A}$, and the estimated focal plane depth of channel $C$ is $d_{C}^{0^{\prime}}$ for $C \in\{A ; B\}$, given by $d_{C}^{0^{\prime}}=$ $x\left(\frac{x}{f_{C}+\gamma_{C}^{\max }}-1\right)^{-1}$. The depth point of interest $\left(d_{n}^{\prime}\right)$ for our method is the point of intersection where the two channels show equal blur radii, yielding $\Delta_{B, A}^{\prime}\left(d_{n}^{\prime}\right)=0$. Depth $d_{n}^{\prime}$, assuming $E_{B, A}$ is not substantially large, is given by

$$
d_{n}^{\prime}=\frac{2 L x}{L\left(\frac{x}{f_{A}+\gamma_{A}^{\max }}+\frac{x}{f_{B}+\gamma_{B}^{\max }}\right)-E_{B, A}-2 L} .
$$

A mistake in region mapping (defined by the sign of $\Delta$ ) can cause the loss of the bijective mapping only when $d_{n}^{\prime}<$ $d_{A}^{0}$ or $d_{n}^{\prime}>d_{B}^{0}$. Shifts resulting in $d_{n}^{\prime} \in\left[d_{A}^{0}, d_{B}^{0}\right]$ preserve a correct one-to-one mapping. This is because both $A$ and $B$ channels have an bijective mapping and either of them can be used in the range $\left[d_{A}^{0}, d_{B}^{0}\right]$. Therefore, discrepancies between $d_{n}$ and $d_{n}^{\prime}$ can be tolerated and do not affect the disambiguation process as long as $d_{n}^{\prime} \in\left[d_{A}^{0}, d_{B}^{0}\right]$.

\section{Experimental Results}

We show that the relationships derived between defocus blur, depth and wavelength extend to a typical lens, and demonstrate the generalization of our proposed measure $\Delta$. PSF magnitude plots are obtained experimentally with a Canon EF 50mm f/2.5 lens for the first two plots (with different focal planes) and with a Canon EF 50mm f/1.8 II lens for the third plot (Fig. 1). For our proposed method to generalize, the main property of our $\Delta$ measure must still hold. Essentially, this means that its value must change signs only once with increasing depth, and exactly in the depth range delimited by the two focal planes corresponding to the chosen channels. It is advantageous to compute $\Delta$ between a color channel and the NIR channel due to the larger differential blur between them. This is due to the larger wavelength separation, and due to the uncorrected chromatic aberration in NIR (done only in superachromatic lenses [4, p. 105]). However, the property of $\Delta$ is valid even between spectrally adjacent channels, namely green and red. The $\Delta$ plots are shown in the bottom row of Fig. 1, where $\Delta_{R, G}$ and $\Delta_{N I R, G}$ are plotted in red and black, respectively. They each change signs correctly between their corresponding focal planes.

\section{References}

1. A. Sellent, and P. Favaro, "Which side of the focal plane are you on?," in IEEE ICCP, pp. 1-8, 2014.

2. P. Trouvé, F. Champagnat, G. Le Besnerais, J. Sabater, T. Avignon, and J. Idier, "Passive depth estimation using chromatic aberration and a depth from defocus approach," in Applied Optics, vol. 52, pp. 7152-7164, Optical Society of America, 2013.

3. S. Bhuo, and T. Sim, "Defocus map estimation from a single image," in Pattern Recognition, pp. 1852-1858, 2011.

4. E. Allen, and S. Triantaphillidou, "The Manual of Photography and Digital Imaging," in CRC Press, 2012. 